\title{
Expression of p53 and PCNA in Cholangiocarcinoma and Primary Sclerosing Cholangitis
}

\author{
Nirmala Batheja, M.D., Arief Suriawinata, M.D., Romil Saxena, M.D., Georgette Ionescu, \\ Myron Schwartz, M.D., Swan N. Thung, M.D. \\ The Lillian and Henry M. Stratton-Hans Popper Department of Pathology and the Division of \\ Hepatobiliary Surgery of the Recanati-Miller Transplant Institute, Mount Sinai School of Medicine, New \\ York, New York.
}

The aim of this study was to identify the pattern and significance of expression of p53 and PCNA in cholangiocarcinoma and primary sclerosing cholangitis. Histological sections from 18 patients with cholangiocarcinoma ( 3 of the cases were associated with primary sclerosing cholangitis), 10 patients with primary sclerosing cholangitis without cholangiocarcinoma, and 7 patients with cirrhosis without cholangiocarcinoma or primary sclerosing cholangitis were stained immunohistochemically for p53 and PCNA. Samples from 17 patients with cholangiocarcinoma (94\%) stained positively for p53. Among these 17 cases, nontumorous bile duct epithelium was positive in 7 (including 3 cases with primary sclerosing cholangitis and 2 with carcinoma in situ), and were positive proliferating bile ductules in 4 cases. The single p53-negative cholangiocarcinoma did not show $\mathbf{p 5 3}$ positivity in either the bile duct epithelium or the proliferating bile ductules. Bile ductal and ductular cells in all 10 patients with primary sclerosing cholangitis without cholangiocarcinoma and in the 7 controls were not reactive for p53. All 18 samples from patients with cholangiocarcinoma $(100 \%)$ were positive for PCNA protein. Bile duct epithelium was positive for PCNA in nine cases $(90 \%)$ of primary sclerosing cholangitis without cholangiocarcinoma and in six (85\%) controls. Our study showed a high rate of p53 expression (94\%) in cholangiocarcinoma. The adjacent uninvolved bile duct epithelium was also immunoreactive for p53 in 7 of 17 patients $(41 \%)$. These findings suggest an early p53 mutation in bile ductal cells in cholangiocarcinogenesis. Expression of 553 may potentially be used to identify or screen, by bile duct brushings, cases of primary sclerosing

Copyright (C) 2000 by The United States and Canadian Academy of Pathology, Inc.

VOL. 13, NO. 12, P. 1265, 2000 Printed in the U.S.A.

Date of acceptance:

Address reprint requests to: Nirmala Batheja, M.D., Department of Pathology, Box 1194, The Mount Sinai School of Medicine, One Gustave L. Levy Place, New York, NY 10029; fax 212-860-7851. cholangitis suspected of harboring cholangiocarcinoma. Expression of PCNA was a universal feature in cholangiocarcinoma.

KEY WORDS: Cholangiocarcinoma, p53, PCNA, Primary sclerosing cholangitis.

Mod Pathol 2000;13(12):1265-1268

Point mutation of p53 tumor suppressor gene, located on Chromosome 17p, represents one of the most common genetic alterations found in human cancers. Immunohistochemical demonstration of p53 is thought to reflect mutations of the p53 gene. p53 expression or mutation has been studied in a variety of cancers, but there have been only a few studies investigating the expression of p53 in cholangiocarcinoma (CC) and primary sclerosing cholangitis (PSC; 1-6). Such studies are particularly important because development of cholangiocarcinoma in PSC is common, varying from $7 \%$ in asymptomatic patients to $42 \%$ in necropsy series (7).

PCNA, also known as cyclin, is a nonhistone, $36-\mathrm{kDa}$ nuclear protein that is involved in the initiation of cell proliferation. Immunostaining of tissue sections with PCNA can be used to assess tumor cell proliferation. There are only rare studies reported that assess the expression and significance of PCNA in cholangiocarcinoma and primary sclerosing cholangitis $(8,9)$.

We embarked on a study to examine the pattern and significance of p53 and PCNA expression in CC and PSC, which represents a well-known risk factor for cholangiocarcinogenesis.

\section{METHODS}

Histological sections from 18 patients with cholangiocarcinoma (associated in 3 cases with PSC and 2 cases with identified carcinoma in situ in bile ducts), 10 patients with PSC without CC, and 7 patients of cirrhosis without CC or PSC were 
stained immunohistochemically for p53 and PCNA by a standard avidin-biotin detection system using antigen retrieval with a citrate buffer at pH 6 .

Four-micron-thick sections of formalin-fixed paraffin-embedded tissue were deparaffinized, rehydrated through graded alcohols, then transferred into $0.01 \mathrm{M}$ citrate buffer ( $\mathrm{pH}$ 6.0), boiled on a hot plate for 20 minutes, cooled for 40 minutes, and washed in distilled water for 5 minutes. Endogenous peroxidase activity was blocked with $3 \%$ hydrogen peroxide for 10 minutes. Sections were then incubated with monoclonal p53 antibody (Clone BP53 12-1, Biogenex, CA) at a dilution of 1:40 for 30 minutes at room temperature or with PCNA antibody (Clone 19 A2, Biogenex) at a dilution of 1:80 for 2 hours at room temperature. Sections were then incubated with a biotinylated rabbit antimouse IgM (Dako E465) diluted 1:80 in PBS for 20 minutes at RT, followed by streptavidin conjugated with horseradish peroxidase substrate, composed of 3,3' diaminobenzidine $0.01 \mathrm{gm}$, Triton solution (0.5\%) $15 \mathrm{~mL}$, and $\mathrm{H}_{2} \mathrm{O}_{2}(30 \%) 0.01 \mathrm{~mL}$, was then applied to sections for 10 minutes. Sections were then stained with hematoxylin and mounted in Permount.

The immunostaining of cells for both p53 and PCNA was assessed by two independent observers and was recorded as positive when nuclei of cells exhibited brown staining. The findings were scored according to the number of positive cells:,$+<25 \%$; ,++ 25 to $50 \%$, and,$+++>50 \%$. Sections of colon adenocarcinoma with p53 $(++)$ positivity were used as positive controls for p53, and sections of tonsils were used as positive controls for PCNA. Negative controls included replacement of primary antibodies to p53 and PCNA with PBS. The location of the tumor in the liver, whether hilar or periph- eral, and the size of tumors were recorded (see Table 1).

\section{RESULTS}

Seventeen out of 18 patients with cholangiocarcinoma (94\%) stained positively for p53 (Fig. 1). The staining was categorized + in $13,++$ in 2 , and +++ in 2 patients (Table 1 ). In 7 of the 17 p53positive patients with $\mathrm{CC}$, uninvolved bile duct epithelium (BD) was also p53 positive, including that from three out of three patients with PSC (Fig. 2) and from two out of two patients with carcinoma-in situ (Table 1). Proliferating bile ductules (PBD; Fig. 3 ) were positive for p53 in four patients, in one case, in association with PSC and in another one, with CIS. The single p53 negative case of cholangiocarcinoma was also negative for p53 in BD and PBD. Bile ductal and ductular epithelial cells were also negative in all 10 patients with PSC without CC and in all 7 cirrhotic controls (Table 2). BD was positive for p53 in all three patients of PSC with CC but was negative in all of the patients with PSC without CC.

All cases of cholangiocarcinoma (100\%) were positive for PCNA, mostly categorized ++ or +++ (Fig. 4). Among these, PCNA was expressed in BD in 13 patients and in PBD in 2 patients (Table 1). BD was positive for PCNA in 9/10 patients with PSC without CC and in 6/7 controls (Table 2).

\section{DISCUSSION}

P53 mutations are known to occur frequently in human cancers, and p53 expression has been studied in a variety of carcinomas, but only few studies have reported the expression of p53 in CC and PSC (1-6). Cell proliferation activity is an important in-

TABLE 1. Staining Pattern of p53 and PCNA in 18 Cases of Cholangiocarcinoma

\begin{tabular}{|c|c|c|c|c|c|c|c|c|c|c|}
\hline & \multirow{2}{*}{$\begin{array}{l}\text { Tumor } \\
\text { Location }\end{array}$} & \multirow{2}{*}{$\begin{array}{c}\text { Tumor } \\
\text { Size }(\mathrm{cm})\end{array}$} & \multirow{2}{*}{$\begin{array}{l}\text { Associated } \\
\text { PSC or CIS }\end{array}$} & \multicolumn{3}{|c|}{ p53 } & \multicolumn{4}{|c|}{ PCNA } \\
\hline & & & & Tumor & Bile Duct & Ductules & Tumor & Bile Duct & Ductules & Peribiliary Gland \\
\hline 1 & $\mathrm{P}$ & 2.5 & - & + & - & - & ++ & - & - & - \\
\hline 2 & $\mathrm{H}$ & 8 & - & + & - & + & +++ & + & - & - \\
\hline 3 & $\mathrm{H}$ & 8 & 3 & + & - & - & +++ & - & - & - \\
\hline 4 & $\mathrm{H}$ & 3 & - & + & - & - & ++ & ++ & - & - \\
\hline 5 & $\mathrm{P}$ & 3 & - & + & + & - & +++ & + & - & - \\
\hline 6 & $\mathrm{P}$ & 7 & PSC & +++ & ++ & ++ & ++ & + & - & - \\
\hline 7 & $\mathrm{P}$ & 9.5 & - & ++ & - & - & + & + & - & - \\
\hline 8 & $\mathrm{H}$ & 0.8 & CIS & + & + & - & +++ & + & - & - \\
\hline 9 & $\mathrm{H}$ & $\mathrm{n} / \mathrm{a}$ & PSC & + & +++ & - & + & + & - & + \\
\hline 10 & $\mathrm{H}$ & 7 & - & + & - & - & ++ & - & - & - \\
\hline 11 & $\mathrm{P}$ & 7.5 & - & + & - & + & +++ & + & - & - \\
\hline 12 & $\mathrm{H}$ & 3 & - & + & + & - & ++ & + & + & + \\
\hline 13 & $\mathrm{H}$ & 0.6 & CIS & +++ & + & + & ++ & + & + & - \\
\hline 14 & $\mathrm{P}$ & 0.8 & - & + & - & - & + & - & - & - \\
\hline 15 & $\mathrm{H}$ & 3.5 & PSC & + & + & - & + & + & - & - \\
\hline 16 & $\mathrm{P}$ & 7.5 & - & ++ & - & - & ++ & - & - & - \\
\hline 17 & $\mathrm{H}$ & 1 & - & - & - & - & ++ & + & - & + \\
\hline 18 & $\mathrm{H}$ & 17 & - & + & - & - & +++ & + & - & - \\
\hline
\end{tabular}

$\mathrm{P}$, peripheral tumor; H, hilar tumor; PSC, primary sclerosing cholangitis; CIS, carcinoma in situ. 


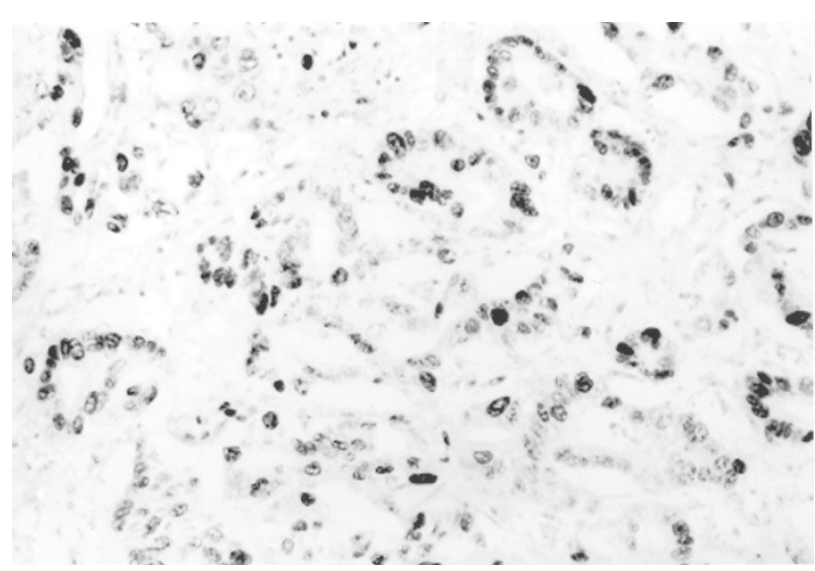

FIGURE 1. Cholangiocarcinoma with positive staining for p53 (immunoperoxidase stain, counterstained with methyl green, 100×).

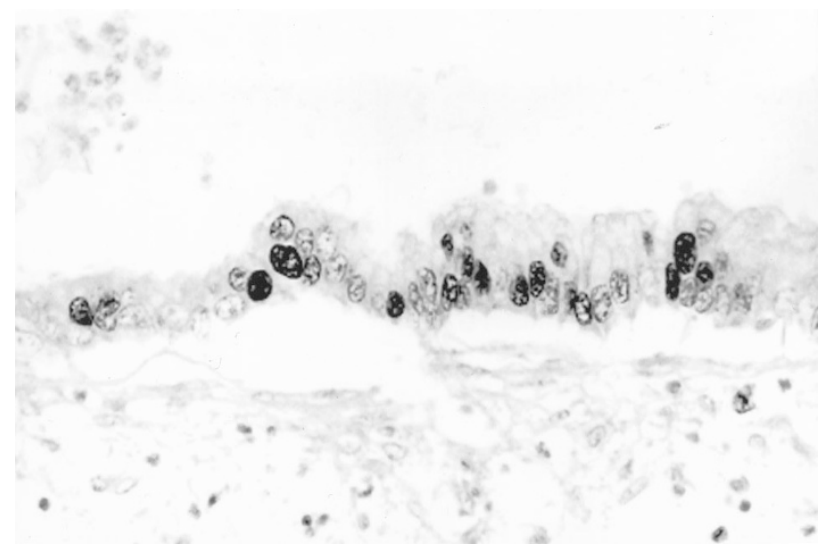

FIGURE 2. Bile ductal epithelium expressing p53 (immunoperoxidase stain, counterstained with methyl green, $400 \times$ ).

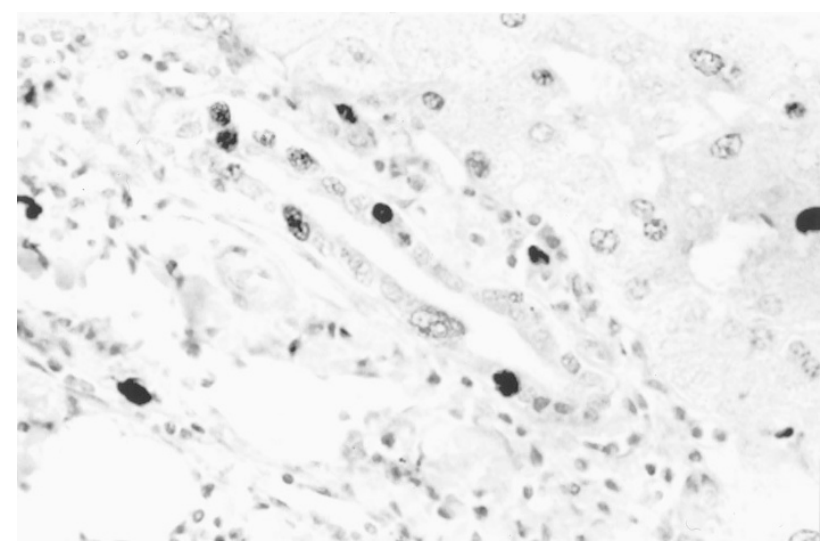

FIGURE 3. P53 in bile ductular cells in a case of primary sclerosing cholangitis harboring cholangiocarcinoma (immunoperoxidase stain, counterstained with methyl green, $200 \times$ ).

dicate of growth and behavior of various human cancers, but only rare studies have reported the expression and significance of PCNA in cholangiocarcinoma $(8,9)$.

Our study showed a high rate of positivity for p53 and PCNA in cholangiocarcinoma. P53 was positive
TABLE 2. Staining Pattern of p53 and PCNA in PSC without Cholangiocarcinoma and Controls

\begin{tabular}{|c|c|c|c|c|}
\hline & \multicolumn{2}{|c|}{$\begin{array}{l}\text { Bile Duct Epithelium } \\
\text { in PSC without } \\
\text { Cholangiocarcinoma }\end{array}$} & \multicolumn{2}{|c|}{$\begin{array}{c}\text { Bile Duct } \\
\text { Epithelium in } \\
\text { Controls (Cirrhosis) }\end{array}$} \\
\hline & p53 & PCNA & p53 & PCNA \\
\hline 1 & - & $++\mathrm{Tc}-$ & - & \\
\hline 2 & - & +++ & - & + \\
\hline 3 & - & - & - & + \\
\hline 4 & - & +++ & - & ++ \\
\hline 5 & - & +++ & - & ++ \\
\hline 6 & - & + & - & + \\
\hline 7 & - & +++ & - & +++ \\
\hline 8 & - & +++ & & \\
\hline 9 & - & +++ & & \\
\hline 10 & - & +++ & & \\
\hline
\end{tabular}

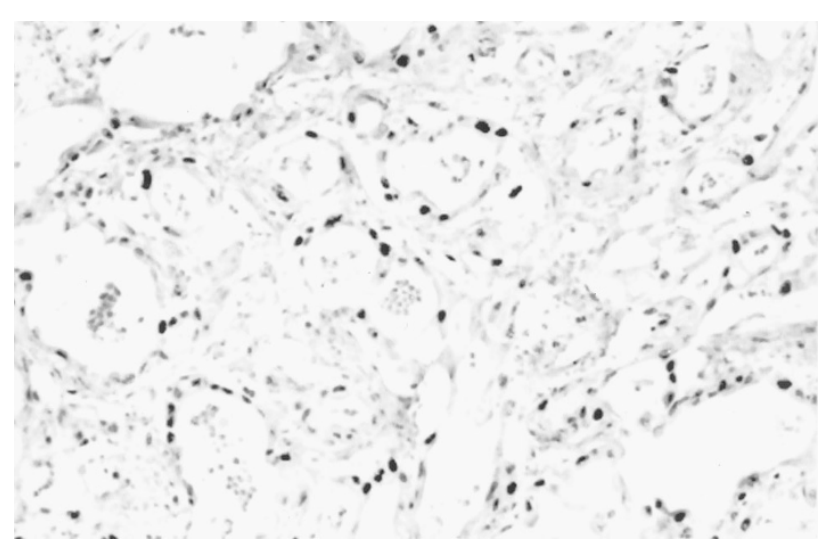

FIGURE 4. Cholangiocarcinoma with positive staining for PCNA (immunoperoxidase stain, counterstained with methyl green, $100 \times$ ).

in bile ducts in all three patients with PSC with CC but was negative in those with PSC without CC and in controls. Expression of p53 in bile ducts of liver with cholangiocarcinoma, especially when PSC was present (three patients) or when associated with carcinoma-in situ (two patients), suggest an early mutation in bile duct epithelium during cholangiocarcinogenesis and may potentially be used to identify or screen, by bile duct brushings, patients with PSC suspected of harboring cholangiocarcinoma. This finding confirms the previously reported usefulness of p53 by Rizzi et al. (2) as an additional criterion for the diagnosis of cholangiocarcinoma development in patients with PSC. Rizzi et al. (2) reported expression of p53 in 11/14 patients with cholangiocarcinoma, 13 of whom had underlying PSC. No p53 protein was detected in 15 patients with PSC but without cholangiocarcinoma.

Expression of PCNA, however, was not only a universal feature in cholangiocarcinoma but was also present in the nonneoplastic bile duct epithelium in patients with PSC without CC and in cirrhotic controls. Therefore, unlike p53, PCNA immunohistochemistry is not significant for early diagnosis of CC. Furthermore, PCNA positivity did 
not correlate with the size of the tumor or with the extent of p53 expression. This is in variance with the previous findings by Yang et al. (8) and Ota et al. (9), who reported a high proliferative activity associated with higher tumor grade and poorer prognosis. Thus, the presence of PCNA-positive cells in the bile duct epithelium in cases of CC, as well as in cases of PSC without CC and in cases of cirrhosis in our study, suggests that PCNA is related to proliferative activity of ductal epithelial cells rather than to its neoplastic transformation.

$\mathrm{K}$-ras mutation is a common and early event in extrahepatic bile duct carcinomas that contrasts with the early p53 mutation in cholangiocarcinoma (10). The genetic mutations in cholangiocarcinoma, on the other hand, are similar to those reported in the gallbladder (11).

In conclusion, the expression of p53, but not PCNA, may be useful in identifying cholangiocarcinoma and/or potential for malignant transformation of cases with high risk of cholangiocarcinogenesis.

\section{REFERENCES}

1. Washington K, Gottfried MR. Expression of p53 in adenocarcinoma of the gallbladder and bile ducts. Liver 1996;16:99104.

2. Rizzi PM, Ryder SD, Portmann B, Ramage JK, Naoumov NH, Williams R. p53 Protein overexpression in cholangiocarcinoma arising in primary sclerosing cholangitis. Gut 1996;38: 265-8.
3. Ohashi K, Nakajima Y, Kanehiro H, Tsutsumi M, Taki J, Aomatsu Y, et al. Ki-ras Mutations and p53 protein expressions in intra-hepatic cholangiocarcinomas: relation to gross tumor morphology. Gastroenterology 1995;109:1612-7.

4. Terada T, Shimizu K, Izumi R, Nakanuma Y. Methods in pathology. p53 expression in formalin-fixed, paraffinembedded archival specimens of intrahepatic cholangiocarcinoma: retrieval of p53 antigenicity by microwave oven heating of tissue sections. Mod Pathol 1994;7:249-52.

5. Roncalli M, Patriarca C, Gambacorta M, Viale G, Coggi G. Expression of new phenotypic markers in cholangiocarcinoma and putative precursor lesions. J Surg Oncol Suppl 1993;3:173-4.

6. Kiba T, Tsuda H, Pairojkul C, Inoue S, Sugimura T, Hirohashi S. Mutations of the p53 tumor suppressor gene and the ras gene family in intrahepatic cholangiocellular carcinomas in Japan and Thailand. Mol Carcinog 1993;8:312-8.

7. Rosen CB, Nagorney DM, Wiesner RH, Coffey RJ Jr; La Russo NF. Cholangiocarcinoma complicating primary sclerosing cholangitis. Ann Surg 1991;213:21-5.

8. Yang Z, Zhong D, Li Y. [The expression and significance of proliferating cell nuclear antigen/cyclin in cholangiocarcinoma and gallbladder carcinoma.] Chung Hua Wai Ko Tsa Chih 1995;33:437-8.

9. Ota H, Kazama K, Yoshida H, Takeda M, Sakagami H. Rat hepatocellular carcinogenesis and proliferating cell nuclear antigen expression induced by 3 '-mehtyl-4-dimethyl aminoazobenzene. In Vivo 1994;8:289-94.

10. Wistuba II, Albores-Saavedra J. Genetic abnormalities involved in the pathogenesis of gallbladder carcinoma. J Hepatobiliary Pancreat Surg 1999;6:237-44.

11. Wistuba II, Sugio K, Hung J, Kishimoto Y, Virmani AK, Roa I, et al. Allele-specific mutations involved in the pathogenesis of endemic gallbladder carcinoma in Chile. Cancer Res 1995; 55:2511-5. 